\title{
Uniformity, Bipolarization and Pluriformity Captured as Generic Stylized Behavior with an Agent-Based Simulation Model of Attitude Change*
}

\author{
WANDER JAGER \\ Faculty of Management and Organisation, University of Groningen, The Netherlands \\ email:w.jager@bdk.rug.nl \\ FRÉDÉRIC AMBLARD \\ Laboratory of Statistical Physics, Ecole Normale Supérieure, Paris, France \\ email: amblard@cemagref.fr
}

\begin{abstract}
This paper focuses at the dynamics of attitude change in large groups. A multi-agent computer simulation has been developed as a tool to study hypothesis we take to study these dynamics. A major extension in comparison to earlier models is that Social Judgment Theory is being formalized to incorporate processes of assimilation and contrast in persuasion processes. Results demonstrate that the attitude structure of agents determines the occurrence of assimilation and contrast effects, which in turn cause a group of agents to reach consensus, to bipolarize, or to develop a number of subgroups sharing the same position. Subsequent experiments demonstrate the robustness of these effects for a different formalization of the social network, and the susceptibility for population size.
\end{abstract}

Keywords: attitude dynamics, Social Judgment Theory, agent based simulation

\section{Introduction}

The spreading of attitudes and opinions through a population is a crucial process in understanding the dynamics of e.g., political changes, shifts in preferences, the rise and fall of interest groups and the like. Whereas the quality of arguments may determine the extend to which one is being persuaded by another person, often people respond quite simple by favoring positions close to their own, and rejecting more distant positions. The Social Judgment Theory (SJT: Sherif and Hovland, 1961) is a theory that describes how individuals change their position after being confronted with another position. The basic idea of this theory is that a change of a person's attitude depends on the position of the persuasive message that is being received. If the advocated position is close to the initial position of the receiver, it is assumed that this position falls within the latitude of acceptance of the receiver. As a result, the receiver is likely to shift in the direction of the advocated position (assimilation).

*This paper won the best paper award at NAACSOS 2004, Pittsburgh PA. NAACSOS is the main conference of the North American Association for Computational Social and Organizational Science. 
If the advocated position is distant to the initial position of the receiver, it is assumed that this position falls within the latitude of rejectance of the receiver. As a result, the receiver is likely to shift away from the advocated position (contrast). If the advocated position falls outside the border of the latitude of acceptance, but is not that distant that it crosses the border of the latitude of rejectance, it will fall within the latitude of non-commitment, and the receiver will not shift its initial position.

Whereas the Social Judgment Theory has been tested extensively for small laboratory settings, empirical work on how assimilation and contrast effects affect attitude change at the population level has not been done due to methodological limitations. Obviously, multiagent simulation provides a methodology capable of exploring dynamics of attitude change in large populations. Several researchers have worked on simulating how opinions, attitudes or voting behavior in groups emerges from locally interacting people, some working on binary opinions (e.g., Latane and Nowak, 1997; Galam, 1999) and some using continuous opinions, where influence is dependant on distance (using a threshold, e.g., Deffuant et al., 2001, 2002; Weisbuch et al., 2002; Hegselmann and Krause, 2002).

This paper is aimed at extending this line of research by formalizing the Social Judgment Theory in a multi-agent computer simulation. Whereas previous models incorporated processes of assimilation, and to a lesser extend, processes of non-commitment, the current paper also adds processes of contrast. This introduces an opposite mechanism in the model that may have significant consequences for the generated dynamical processes.

A next extension of the model will focus on the importance of attitudes, which Social Judgment Theory addresses as "ego-involvement". The basic assumption here is that ego-involvement provides an important anchor for a person's attitude on an issue. Ego-involvement is being formalized as the distance between one's own initial position and the borders for the latitude of rejection. The higher one's ego-involvement is, the closer the borders to one's own position, and hence the smaller the latitude of non-commitment gets. In a crisis situation, the latitude of non-commitment virtually disappears, and the person either accepts or rejects any attitude toward the topic (O'Keefe, 1990).

\section{Proposed Model}

We have a population with $N$ individuals. Each individual $i$ has got an opinion (an attitude) $x_{i}$, a threshold determining the latitude of acceptance $u_{i}$ and a threshold determining the latitude of rejection $t_{i}$ with $t_{i}>u_{i}$. Varying the values of $t_{i}$ and $u_{i}$ allows for modeling agents having different attitude structures. For example, an agent having a high ego-involvement can be formalized as an agent where $t_{i}$ is slightly larger or equal to $u_{i}$. The agents are scheduled to communicate on a random basis by scheduling random pairs for each timestep of the simulation. During the interaction between individual $i$ and individual $j$, the following rules are applied:

$$
\begin{array}{rl}
\text { If }\left|x_{i}-x_{j}\right|<u_{i} & d x_{i}=\mu \cdot\left(x_{j}-x_{i}\right) \\
\text { If }\left|x_{i}-x_{j}\right|>t_{i} & d x_{i}=\mu \cdot\left(x_{i}-x_{j}\right)
\end{array}
$$

where the parameter $\mu$ controls for the strength of influence. 
The same rules are applied for the update of the opinion of the individual $j$.We will first study the simplest case of this simple model. The initial conditions in the simulations are the following: The opinions are drawn from a uniform distribution between $[-1 ; 1]$. Individuals are initialized with the same uncertainty $U$ and the same threshold $T$. Individuals are fully connected or full mixed, so every individual from the population can interact with any other. Interactions will take place between randomly selected pairs.

\section{Attitude Dynamics with Random Contacts Between Agents}

In the first experiment we vary the values of $U$ and $T$ between 0.1 and the maximum of 2.0 , increasing the values stepwise by 0.1 , with the constraint that $T>U$. The resulting conditions are being run with 400 agents, setting the speed of the dynamics $\mu$ at 0.1 .

Figure 1 shows that different settings of $U$ and $T$ result in the emergence of 1,2, 3 or more groups sharing the same opinion. In the following we present some typical runs for different conditions for the values of $T$ and $U$.

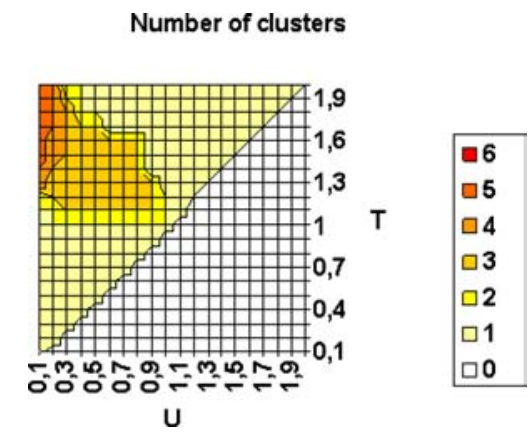

Figure 1. Overview of the average number of clusters at the end of simulation experiments for $U$ varying between 0.1 and 2.0 and $T$ varying between 0.1 and 2.0 (with the constraint $T>U$ ) for 10 replications of each tested couple $(U, T)$.

\subsection{High Ego-Involvement}

In this condition we formalize agents having a relative high ego-involvement, by setting $U$ at 0.4 and $T$ at 0.6 . A characteristic trajectory of the opinions is pictured in figure 2 .

The results show the emergence of a bipolarization of attitude positions. Within this condition the contrast effect dominates the attitude dynamics; because agents have small latitude of acceptance, a random contact has a large chance of eliciting a contrast effect in the agent. The closer an agent gets to one of the extremes, the more likely it is that a random contact will result in (1) a contrasting effect when the other has a more average position, or (2) assimilation if the other is also close to the extreme. 


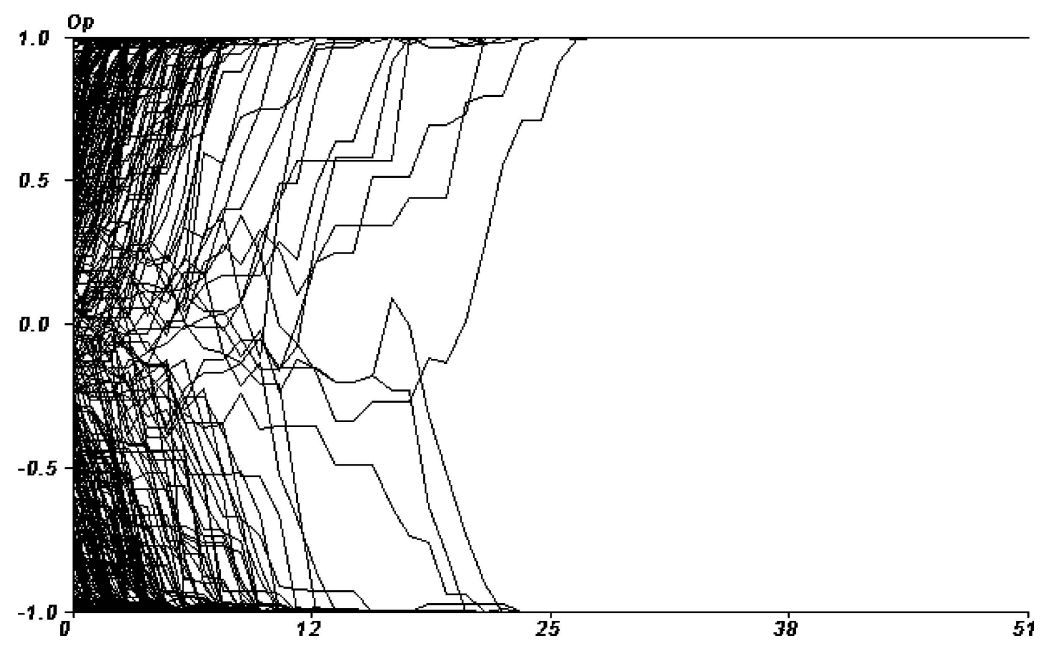

Figure 2. A typical attitude trajectory for agents with $U=0.4, T=0.6$.

\subsection{High Latitude of Acceptance and Non-Commitment}

In the next condition we formalize agents having much higher latitude of acceptance and non-commitment by setting $U$ at 1.2 and $T$ at 1.6. A typical trajectory of the opinions is pictured in figure 3 .

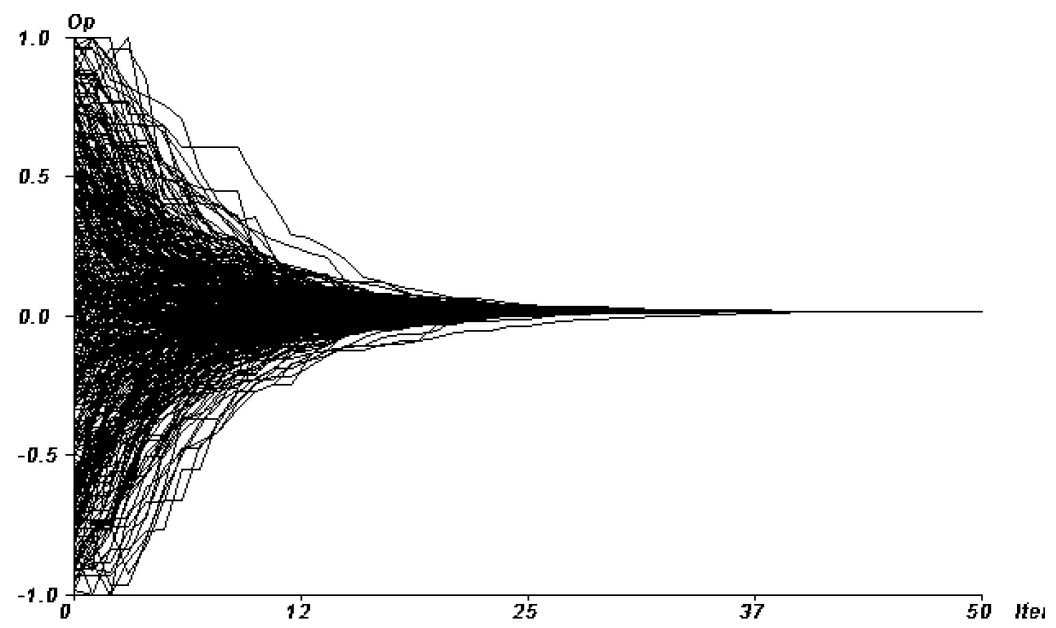

Figure 3. A typical attitude trajectory for agents with $U=1.2, T=1.6$.

We observe that the agents find consensus concerning their attitude position. Within this condition the assimilation effect dominates the attitude dynamics. This is caused by fact that random contacts have a larger chance of falling in the latitude of acceptance than the 
latitude of rejection, causing the agents to assimilate the others' position. As a consequence, there is a strong tendency in the population to move towards each other's attitude position, which clearly results in the group finding a position on the average attitude position.

\subsection{Small Latitude of Acceptance and a High Latitude of Non-Commitment}

In the next condition we formalize agents having relative small latitude of acceptance and large latitude of non-commitment by setting $U$ at 0.6 and $T$ at 1.2.A typical trajectory of the opinions is pictured in figure 4 .

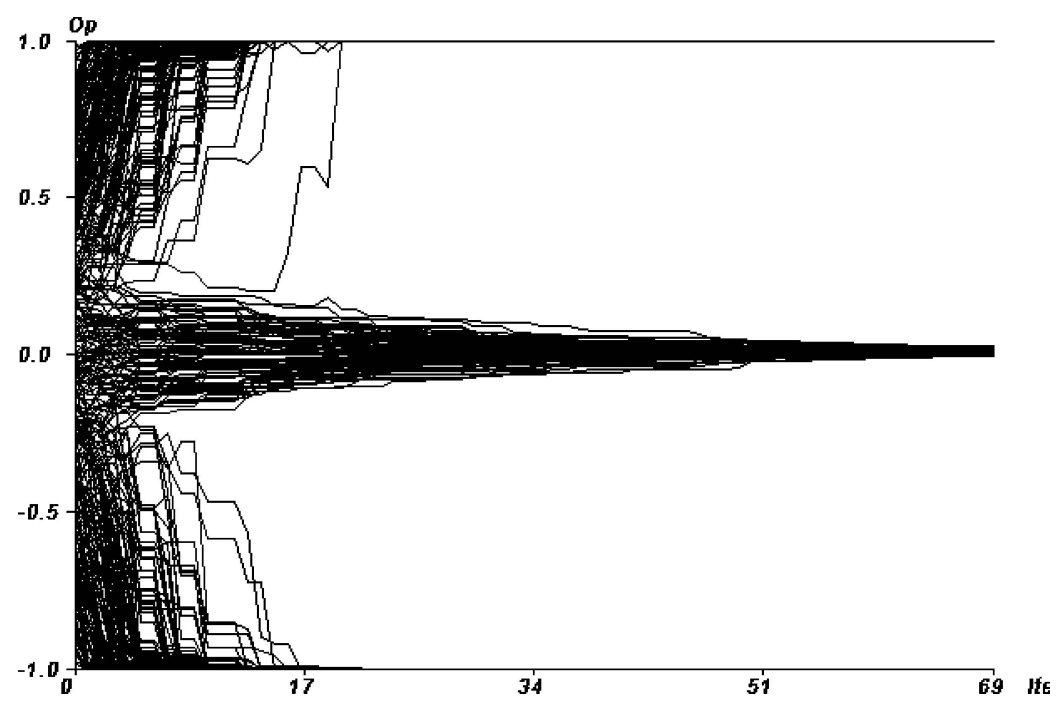

Figure 4. A typical attitude trajectory for agents having $U=0.6, T=1.2$.

In this condition we observe both the contrast and assimilation dynamics. Agents having an initial attitude significantly differing from the average have a larger chance of interacting with an agent having a position in the direction of the average. Hence, they will often respond with a contrast, moving away from the center. On the contrary, agents with an initial about average opinion will contrast themselves with extreme agents from both sides, which results in a balancing of contrast effects. This causes the assimilation effects within this group to converge towards a single position.

\subsection{Very Small Latitude of Acceptance and a Very High Latitude of Non-Commitment}

In the next condition we replicate the previous experiment, only with decreasing the latitude of acceptance $U$ at 0.2 and increasing the latitude of non-commitment $T$ at 1.6. A typical trajectory of the opinions is pictured in figure 5 .

Here, we observe the emergence of five groups, two of them on the extreme positions, one group in the middle, and two groups at respectively 0.4 and -0.4 . The emergence of the 


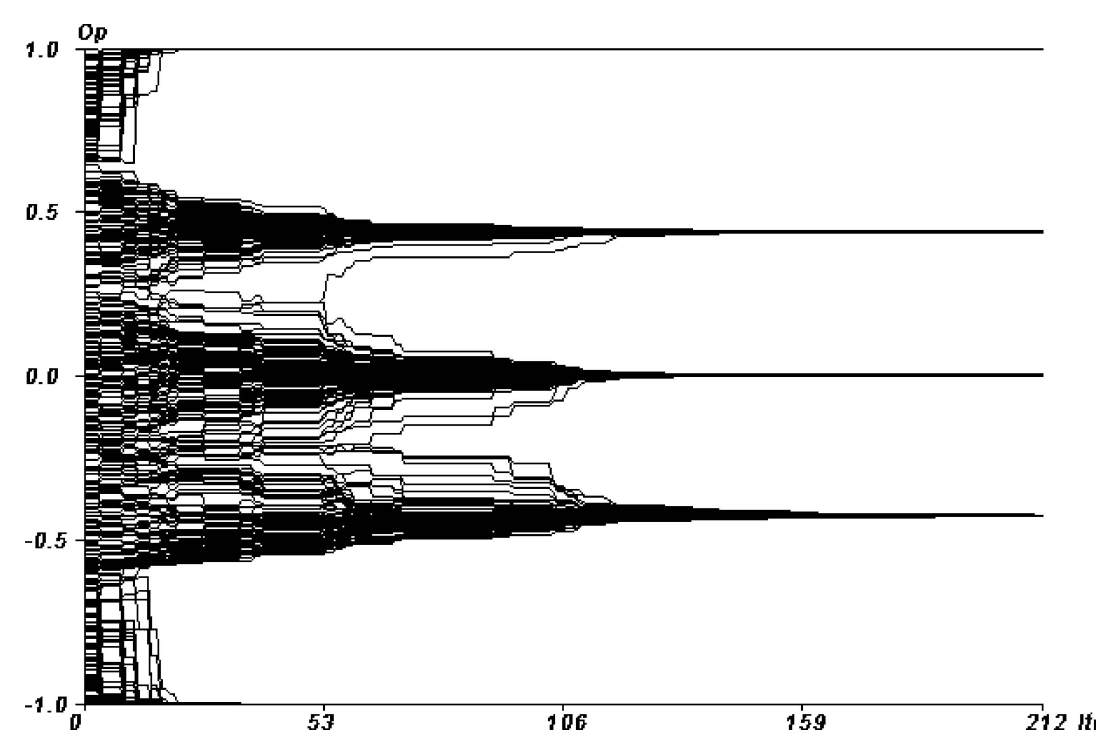

Figure 5. A typical attitude trajectory for agents having $U=0.2, T=1.6$. Colors have no particular meaning in this case.

extreme and central groups can be explained according to the explanation of experiment 4 . More interesting here is the emergence of the two groups at position 0.4 and -0.4 . This can be explained by the fact that for these groups the position of the other groups (including the extremes) wall within the latitude of non-commitment. Hence, only contacts within close range lead towards assimilation effects, causing these groups to converge. Under this condition it is not possible to converge at, e.g., 0.7 or -0.7 , as in these conditions the opposite extreme would cause contrast effects to emerge, and force agents in this position to move towards the adjacent extreme.

\section{Attitude Dynamics with Local Social Networks}

Whereas in the previous series of experiments the agents interacted on a random basis, in real life people are more likely to interact with people belonging to their social network. In a first attempt to study how social networks may affect the qualitative behavior of the model, we decided to replicate the previous experiment using a regular grid as a social network. Using a Von Neumann neighborhood each agent can interact only with the four direct neighbors: North, South, East and West, thus constituting a local network. Figure 6 shows how attitudes evolve over time (presented in rows) for 3 conditions.

In this experiment we observe that the model is not sensitive to the brutal reduction of the average connectivity per agent, going from the totally connected case to a connectivity of four per agent. This behavior of the model is quite rare for a model, as in other cases the introduction of social networks results in an increase of the clustering in 

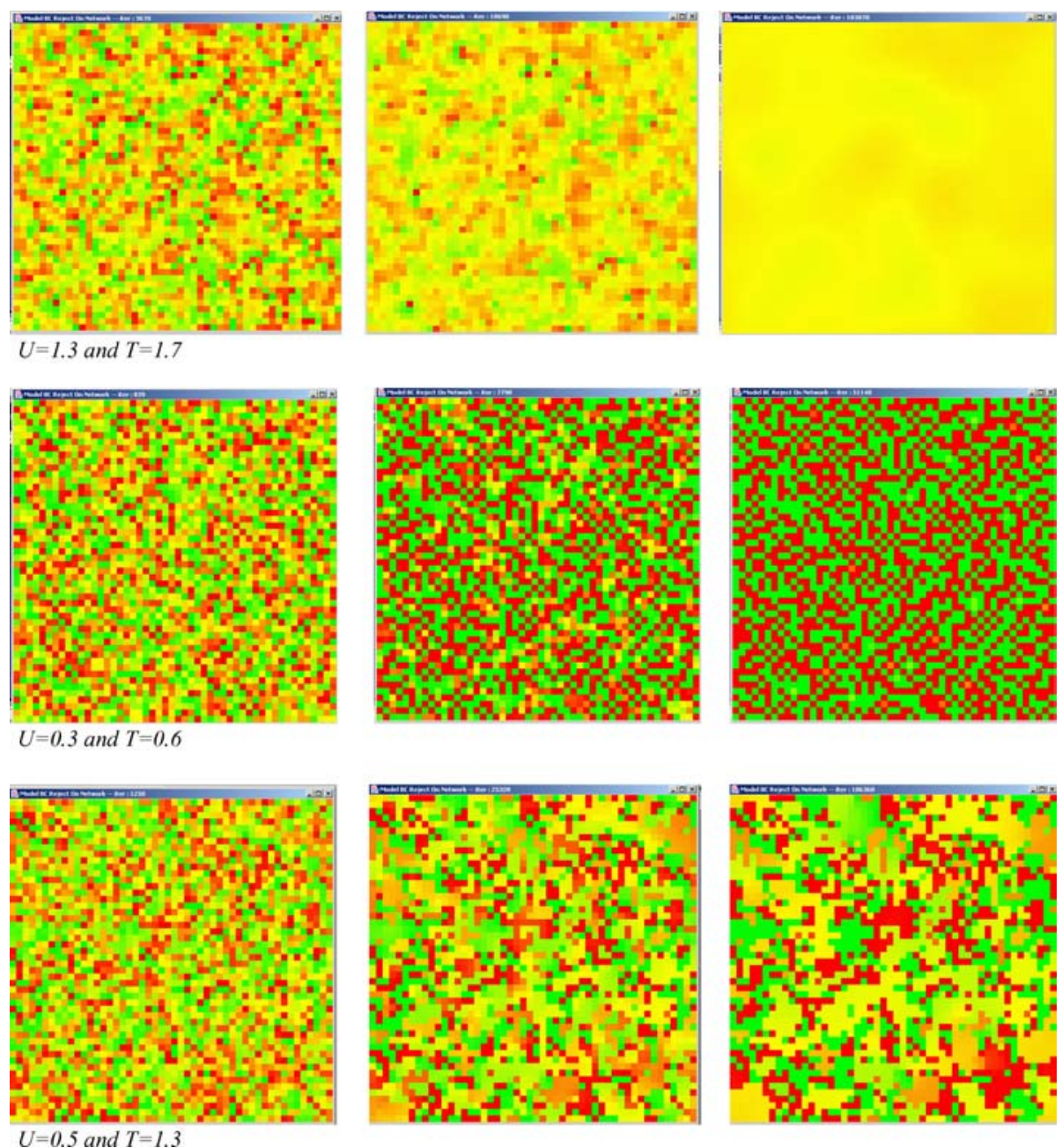

Figure 6. Qualitative results with a local social network, we observe again the three typical cases observed in the full mixed case, time going from left to right, each row corresponding to a particular simulation. On each grid, the color figures the opinion of the agent between -1 (red) and +1 (green) yellow coding for opinions near 0 . Namely (a) Uniformity for $U=1.3$ and $T=1.7$ (b) Bipolarization for $U=0.3$ and $T=0.6$ and (c) Pluriformity for $U=0.5$ and $T=1.3$.

the population (Weisbuch et al., 2002; Latane and Nowak, 1997). This characteristic of the model leads to the conclusion that the three stylized facts we aim to model, namely uniformity, bipolarization and pluriformity, can be obtained using just a few possibilities to interact. These stylized facts at the global scale are then robust to the introduction of social networks, and can be considered as generic results considering the simplicity of the model. 


\section{Effects of Population Size}

In a next experiment we wanted to test if the population size has an effect on the emergence of the stylized facts. Therefore we replicated the condition leading towards uniformity ( $U=1.3$ and $T=1.7$ ), only instead we increased the number of agents from 2500 to 10.000. In figure 7 it can be seen that instead of reaching uniformity, the population shows different attitudes, and the attitude positions change smoothly moving from one area to another. Only a few dissonant agents can be seen in the red and green areas.
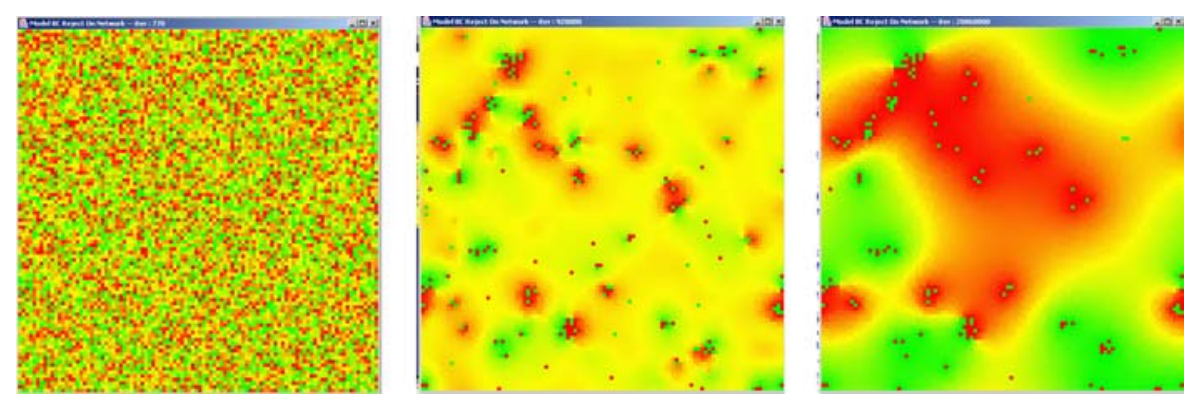

Figure 7. Replication of the $U=1.3$ and $T=1.7$ condition (section 4) with 10.000 agents.

This experiment demonstrates that the stylized facts are susceptible to variations in population size. The more distant agents are, the more likely it is that certain areas may shift in a different attitudinal direction. Whereas in general the spatial transition from green to red shows a smooth path, demonstrating a pluriformity of attitudes, it can also be observed that in the most extreme attitude areas small numbers of dissidents show up. Here a sharp polarization effect emerges on the very local level. Hence the stylized facts may operate simultaneously on different scale levels.

\section{Conclusions}

The first simple experiments demonstrate that differences in ego-involvement may generate different attitude dynamics in the population. In a crisis situation, where the latitude of non-commitment is very small and the ego-involvement is high (as described by O'Keefe, 1990, and represented in the first experiment) we observe that the attitude dynamics leads towards a polarization. On the contrary, when ego-involvement is low and the latitude of non-commitment is very large, we observe the attitude dynamics to generate multiple groups sharing the same attitude position.

In subsequent work we first plan to further investigate the parameter space as to explore the attitude dynamics for homogeneous populations. Here we will focus on different values of $U, T$, the number of contacts and the population size. Extensions of the model will focus on three issues. First we plan to study the effect of heterogeneity of ego-involvement to explore what type of agents has the largest influence on the attitude dynamics. Next we plan to formalize different social networks (small-world and scale-free networks), allowing 
testing how attitude dynamics depends on existing social structures within a population. Formalizing a network where the links between agents depend on similarity of attitude position may provide a flexible network allowing for the testing of group polarization and risky shift phenomena. Finally, we intend to extend the model with multiple attitude issues. This would allow for studying more complex attitude dynamics depending on the salience of different attitudes at a given moment, involving both the emergence and destabilization of groups.

\section{Acknowledgment}

Frédéric Amblard thanks especially Gérard Weisbuch and Guillaume Deffuant for their always valuable support.

\section{References}

Deffuant, G., D. Neau, F. Amblard and G. Weisbuch (2001), “Mixing Beliefs Among Interacting Agents,” Advances in Complex Systems, 3, 87-98.

Deffuant, G., F. Amblard, G. Weisbuch and T. Faure (2002), "How can Extremism Prevail? A Study Based on the Relative Agreement Interaction Model," Journal of Artificial Societies and Social Simulation, 5(4), $<$ http://jasss.soc.surrey.ac.uk/5/4/1.html>

Galam, S. (1999), “Application of Statistical Physics to Politics,” Physica A, 274, 132-139.

Hegselmann, R. and U. Krause (2002), "Opinion Dynamics and Bounded Confidence Models, Analysis and Simulation,” Journal of Artificial Societies and Social Simulation, 5(3), <http://jasss.soc.surrey.ac.uk/5/3/2.html>

Latane, B. and A. Nowak (1997), "Self-Organizing Social Systems: Necessary and Sufficient Conditions for the Emergence of Clustering, Consolidation, and Continuing Diversity," in G.A. Barnett and F.J. Boster (Eds.) Progress in Communication Sciences, Ablex Publishing Corporation.

O'Keefe, D.J. (1990), Persuasion Theory and Research. Sage Publishing, Newbury Park, California. Sherif, M. and C.I. Hovland (1961). Social Judgment. Yale University Press, New Haven, CT.

Weisbuch, G., G. Deffuant, F. Amblard, F. and J.-P. Nadal (2002), "Meet, Discuss and Segregate!,” Complexity, 7(3), 55-63.

Wander Jager received his Ph.D. degree in Social Sciences in 2000 from the University of Groningen, the Netherlands. Dr. Jager is currently Associate Professor at the University of Groningen. His current application domain concerns marketing, innovation diffusion and social simulation. Dr. Jager has authored or co-authored various papers on market dynamics, diffusion processes, resource use and sustainable consumption.

Frédéric Amblard received his Ph.D. degree in Multi-Agent Simulation in 2003 from Blaise Pascal University, Clermont-Ferrand, France. Dr. Amblard is currently Associate Professor at the University of Social Sciences in Toulouse and researcher associated to the CNRS-IRIT, Institute of Research in Computer Sciences in Toulouse. His current application domain now concerns Agent-Based Social Simulation. Dr. Amblard has authored or coauthored various research papers either in computer sciences, in physics or in sociology.

*A preceding version of this paper has been presented to the 2004 Conference of the North American Association for Computational Social and Organization Science, Pittsburgh, USA and received the best paper award from this conference. 